\title{
Accuracy of radial-velocity measurements for early-type stars
}

\section{Spectral-type mismatch in A-type synthetic spectra}

\author{
W. Verschueren ${ }^{\star}$, M. David, and R.E.M. Griffin ${ }^{\star \star}$ \\ Astrophysics Research Group, University of Antwerp (RUCA), Groenenborgerlaan 171, B-2020 Antwerpen, Belgium
}

Received April 29; accepted August 4, 1999

\begin{abstract}
Measuring accurate radial velocities of rotating early-type (O-B-A) stars using cross-correlation techniques is hampered by the fact that object-template spectrum mismatch causes systematic errors that do not cancel out sufficiently in these spectra. This series of papers aims at quantifying those mismatch errors, understanding their astrophysical origin, and developing strategies to avoid them maximally. In this first paper, we employ synthetic spectra to study mismatch between A-type main-sequence stars caused solely by differences in $T_{\text {eff }}$ and $\log g$. We show that this spectral-type mismatch varies greatly throughout the spectrum, though with some degree of systematic dependence on rotational velocity and, to a lesser degree, on temperature. We propose a scheme for selecting spectral regions that should provide, for main-sequence A-type stars, accuracies better than $1 \mathrm{~km} \mathrm{~s}^{-1}$ for $v \sin i \leq 150 \mathrm{~km} \mathrm{~s}^{-1}$ and between $1-2 \mathrm{~km} \mathrm{~s}^{-1}$ for $v \sin i$ up to $300 \mathrm{~km} \mathrm{~s}^{-1}$. The scheme includes sufficient spectral information to keep random errors conveniently small, but excludes all wavelength sub-intervals which produce systematic errors much larger than the above mentioned accuracy. Our predictions confirm the success of the methodology of Fekel $(1985,1999)$. We conclude that the proposed scheme needs further testing on a broad sample of real A-type spectra to see under what conditions of stellar individuality it may break down.
\end{abstract}

Key words: methods: numerical — techniques: radial velocities - techniques: spectroscopic - stars: early-type — stars: kinematics

Send offprint requests to: W. Verschueren,

e-mail: verschue@ruca.ua.ac.be

* Postdoctoral Fellow of the Fund for Scientific Research Flanders (Belgium) (F.W.O.).

** Visiting Professor, RUCA.

\section{Introduction}

Techniques to determine radial velocities (RVs) of stars by measuring the Doppler shifts of their spectral lines have continuously improved throughout the last half-century. Cross-correlation techniques that use optical templates (e.g. Baranne et al. 1979) or numerical ones (e.g. Scarfe et al. 1990; Latham 1992; Baranne et al. 1996) are particulary efficient in that they combine all spectral information and therefore optimize the measuring precision (random errors). There is, however, no guarantee that the accuracy (systematic errors) is optimized as well in this way. Cross-correlations of spectra give rise to systematic errors through "spectrum mismatch" between object and template spectrum. More specifically, such errors arise from asymmetrical differences, when (for example) the components of a blended line do not have the same relative strength in both spectra. In fact, spectrum mismatch is all but inevitable except in the singular case when both spectra arise from the same non-variable star (and provided that neither the observations nor the data reduction process introduced significant differential error).

Spectrum mismatch is caused by differences in atmospheric parameters ( $T_{\text {eff }}, \log g$, abundances), rotational velocity, atmospheric velocity fields (e.g. convection, wind, pulsation), and all peculiarities which individual stars or specific groups of stars may exhibit (e.g. magnetic-field structure). If the template is a synthetic spectrum, the amount of spectrum mismatch will obviously depend on the degree of sophistication included in the model, on the accuracy of the line data, and on the amount of information one has about the object; even the best presentday spectrum synthesis does not simulate all the fine details of an observed spectrum. Incidentally, if RVs are determined not by cross-correlating spectra but by measuring the centroids of individual features, spectrum mismatch causes small wavelength offsets in those measured centroids.

For most categories of late-type (F-G-K) stars, radial velocities are routinely obtained nowadays with precisions 
and accuracies of the order of $0.1-1 \mathrm{~km} \mathrm{~s}^{-1}$. That success derives from intrinsic characteristics of late-type spectra, namely a high density of lines, small line widths, and lines which generally bear a family resemblance to one another over a wide range of spectral types. Thus, one can achieve small random errors from spectra with relatively low signal-to-noise ratio $(S / N)$ and with a small wavelength coverage. Furthermore, systematic mismatch errors between those spectra are likely to be much smaller than $1 \mathrm{~km} \mathrm{~s}^{-1}$ because statistically the effects of the numerous individual blends are approximately evenly distributed in sign and so have a very small nett effect. The same template spectrum can therefore be used for all those latetype stars without the risk of systematic errors, caused by object-template mismatch, larger than quoted above. The latter has facilitated not only the derivation of accurate relative $\mathrm{RVs}$ of different late-type stars, but also the definition of a late-type RV standard star system of which the absolute zero-point could be tied to the Sun the only star whose RV can be measured very accurately independently of its spectrum (Petrie 1962). Nevertheless, it has recently become clear that, even for late-type stars, RV errors within the aforementioned range are dominated at least in some cases by object-template mismatch; this is instanced by the zero-point offset for the RVs of red stars measured with CORAVEL (Stefanik 1997). Convective line shifts may also introduce systematic offsets of several tenths of a $\mathrm{kms}^{-1}$ between different late-type stars (Dravins 1985). It is also well known that the quoted accuracies cannot be obtained routinely for rotating or very peculiar late-type stars, nor for M-type stars, because of the lack of suitable template spectra.

In terms of absolute (random and systematic) errors, the situation is much worse for early-type (O-B-A) stars. A discussion of the quantification and minimization of random errors that arise when cross-correlating early-type spectra has been given by Verschueren \& David (1999). The present series of papers undertakes the (harder) task of addressing systematic errors, which are in general much larger than for late-type stars for the following reasons. The total range of temperature spanned by O-B-A stars is much larger than that encompassed by F-G-K stars, and the relative strengths of the components of many blends vary faster from one sub-class to the next. The low number of available lines produces two opposing effects: blending is less likely, but so is also the chance that mismatch errors will cancel statistically. But the advantage of fewer blends is largely negated when a large rotational velocity is present. The occurrence of broad and strong $\mathrm{H}$ and He lines, while offering possibilities for measuring RVs of rapidly rotating stars, presents other specific problems because of blending and spectrum rectification. In comparison with stars of later types, a disproportionate number of early-type stars exhibits spectral peculiarities that tend to be dominated by the behaviour of certain elements or ions (as in Bp and Ap stars). In stars of the earliest spectral types, some lines are formed in atmospheric velocity fields as large as several $\mathrm{km} \mathrm{s}^{-1}$ (e.g. Ebbets 1979). It will therefore be appreciated that RV measurements of early-type stars are prone to systematic errors that can amount to several $\mathrm{km} \mathrm{s}^{-1}$. Furthermore, they cannot be anchored to the late-type absolute zero-point in a simple way. There has therefore been little progress in defining a system of early-type RV standard stars (see Latham \& Stefanik 1992); the lack of early-type stars with RVs known accurately from spectrum-independent measurements is an additional drawback.

A reduction in error below the $1 \mathrm{~km} \mathrm{~s}^{-1}$ level for the RVs of early-type stars is important for a variety of astrophysical studies, such as the kinematics and dynamics of young stellar groups, duplicity among early-type stars, and correction of high-precision proper motions for perspective acceleration. A general conclusion which we draw from previous studies (see Sect. 2.1) is that a significant improvement in the accuracy of relative RV measurements of different early-type stars, especially in the presence of rotation, may only be achieved by a more "microscopic" approach of identifying and eliminating spectrum mismatch. It is evident that current efforts to establish a system of early-type RV standards to compute absolute RVs (Stefanik 1997; Fekel 1999) require the development of techniques that will specifically avoid spectraltype dependent relative errors (see Verschueren 1995).

In this series of papers, we address questions such as: How large are the errors introduced by cross-correlating spectra of different types of stars between which different varieties of mismatch are likely to occur? Which spectral regions are more favourable than others? To what extent can accuracy be optimized by eliminating unreliable spectral regions? For a given wavelength region selected to obtain a sufficiently small systematic error, what $S / N$ is needed to obtain a sufficiently small random error?

In this first paper we seek a better understanding of the relation between spectrum mismatch caused solely by given differences in $T_{\text {eff }}$ and $\log g$ (hereafter referred to as "spectral-type mismatch"), and the consequent RV error (hereafter referred to as "mismatch shift"). This type of mismatch is all pervasive and is probably the main source of systematic errors. We conduct the experiments with synthetic spectra in order to isolate the temperature/gravity mismatch from additional sources of mismatch not reproduced by the models. The latter will be handled separately, on the basis of purely observational spectra, in subsequent papers. Further advantages of the use of synthetic spectra are given in Sect. 2.3. In this paper, we restrict the investigation to A-type stars on the main-sequence, providing a smooth connection to RV measurements of late-type stars.

Section 2 summarizes previous work, gives an example of the problems we are dealing with and outlines the methodology of our approach. Section 3 describes in detail our experimental set-up and the parameter-space covered. 
The mismatch shifts arising from small individual spectral regions are presented and discussed in Sect. 4. Section 5 proposes possible strategies for the selection and combination of spectral regions to minimize systematic errors, taking also into account the effects of $S / N$ which are inevitable in observed spectra. Section 6 lists our conclusions and proposes future work.

\section{Methodology}

\subsection{Previous work}

The literature describes several systematic approaches for improving the accuracy of early-type RV measurements. Andersen \& Nordström (1983) examined the suitability of individual spectral features in rotating late B- to Ftype stars, basing their study on photographic spectra at $20 \AA \mathrm{mm}^{-1}$. Measurements of individual lines indicated that many lines are affected by errors of up to $10 \mathrm{~km} \mathrm{~s}^{-1}$, depending on spectral-type and rotation. The rms error of a single measurement of any one of their most reliable lines is $3-5 \mathrm{~km} \mathrm{~s}^{-1}$, averaged over all stars, and a set of effective wavelengths was proposed for those lines, appropriate for the ranges of temperature and rotational velocity considered. Their results cannot easily be generalised or compared to ours because of the dependence of those wavelengths on resolution, because of the very different sensitivity to spectrum mismatch of their line-centering technique compared to cross-correlation, and because the wavelength regions we tested (Sect. 3.4) always contain much more spectral information.

A scheme to reduce the effects of mismatch in crosscorrelations, by incorporating a set of observed early-type template spectra of different spectral types (and small rotational velocity), was developed systematically by Liu et al. (1989). A problem in using observed templates is of course that the stars selected may be RV variables, or that their actual velocities may not be known with sufficient accuracy. Liu et al. selected 19 B2- A2 stars with presumed constant RVs and determined their relative RVs by mutual cross-correlation, using a region $150 \AA$ wide around $\mathrm{H} \delta$; more weight was given to results derived from adjacent spectral-types. That template grid was then tied to the late-type absolute zero-point in one of two ways: by crosscorrelating the coolest member of their early-type set with a late-type RV standard, or by demanding that the mean of all velocities agree with the mean of all published velocities for those stars. The accuracy of their system seems to be $\sim 2 \mathrm{kms}^{-1}$, as judged from the difference in zeropoint yielded by their two absolute calibration methods, from their observations of early-type stars in the Pleiades (Liu et al. 1991a, 1991b), and from independent measurements of the latter stars by Morse et al. (1991). Owing to a sliding of the zero-point through the spectral sequence, errors are probably dependent on spectral-type. We argue below that a significant improvement in accuracy may be obtained by using a denser grid of templates, and by crosscorrelating selected spectral regions.

Morse et al. (1991) were the first to introduce the concept of a dense synthetic template grid of temperature and rotational velocity. Their models were based on Kurucz (1979) model atmospheres, from which they selected 2 spectral regions that are dominated by Balmer lines. Zero-point sliding through the grid, caused by the varying spectrum mismatch between synthetic and real spectra, was minimised by quite strong low-frequency fourier filtering of the spectra prior to cross-correlation. The degree of filtering was determined by inspection of the cross-correlation peak, and by demanding that their derived RVs of a set of frequently and carefully observed stars in the range early B to early A agree with their published values (mostly from Fekel 1985) to within $\sim 1 \mathrm{kms}^{-1}$. By applying their method to observations of mid B- to late A-type stars in the Pleiades and $\alpha$ Persei clusters, Morse et al. demonstrated that the early-type zero-point defined by their template/filter grid agreed on average with the late-type absolute zero-point to within $\sim 1 \mathrm{~km} \mathrm{~s}^{-1}$. Although the method was judged successful, anchoring a template grid to a consistent zero-point by the "right" amount of filtering is nevertheless a casual result rather than a purposeful elimination of particular mismatches (see e.g. Verschueren 1991 for experiments with low-frequency filtering to handle mismatch between spectra). A more physically justified approach may lead to an even better and more robust accuracy.

Although it does not deal with early-type stars, the study by Nordström et al. (1994) should be mentioned in this context since it incorporated closely matching synthetic templates for rotating F-type stars. Using the wavelength region $5166-5211 \AA$, they found errors of up to $3 \mathrm{~km} \mathrm{~s}^{-1}$ for $v \sin i \leq 100 \mathrm{~km} \mathrm{~s}^{-1}$, although it is not clear how much was due to systematic errors and how much to random ones. They further found zero-point offsets of up to $\pm 1 \mathrm{~km} \mathrm{~s}^{-1}$ between their results and those from CORAVEL for a variety of rotational velocities up to $50 \mathrm{~km} \mathrm{~s}^{-1}$.

Finally, the most successful (and ongoing) project in this field is that of Fekel $(1985,1999)$ who is monitoring several tens of candidate RV standard stars in the range B2-F2 in order to eliminate those with variable RV. By using relatively isolated $\mathrm{Mg}$ II, Fe II and Ti II lines in the wavelength region $4460-4550 \AA$, and by selecting stars with $v \sin i<50 \mathrm{~km} \mathrm{~s}^{-1}$, Fekel is able to employ only 2 template spectra, the absolute RVs of which were determined by cross-correlation with late F-type standards. Morse et al. (1991) provide indirect evidence that Fekel's RVs are consistent with the late-type zero-point to within $\sim 1 \mathrm{~km} \mathrm{~s}^{-1}$ without spectral-type dependence. Using the most recent velocities of Fekel (1999), we recomputed the average difference and rms spread between Morse et al. 

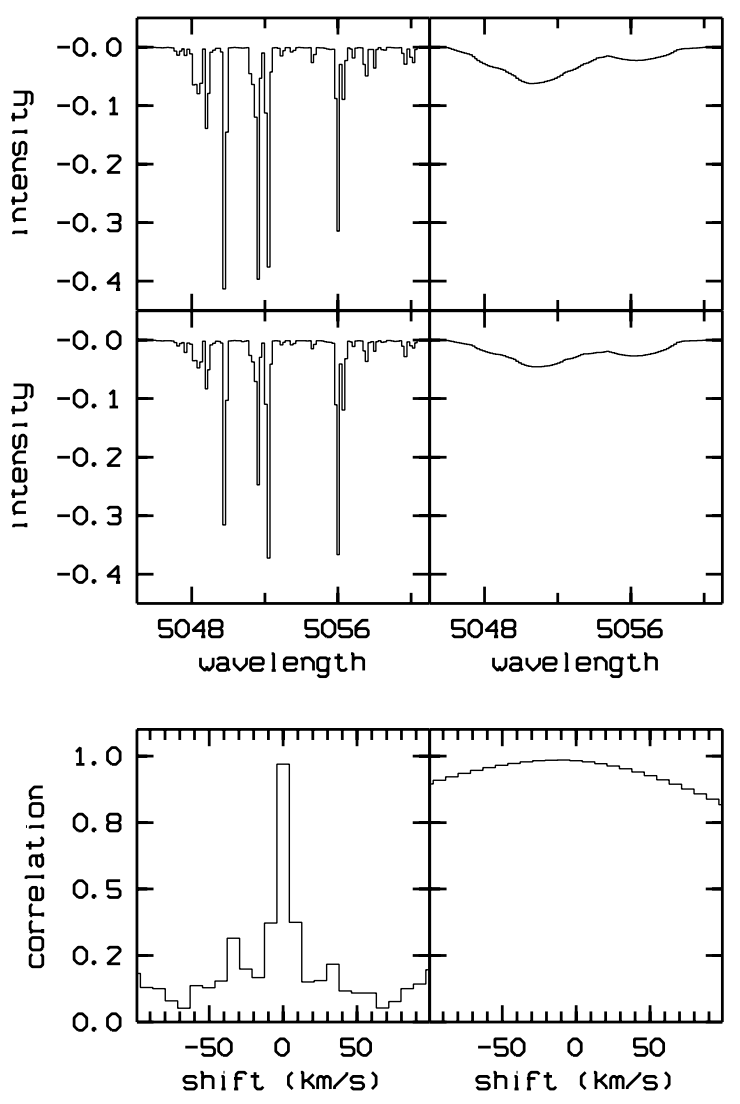

Fig. 1. Top panel: wavelength region of the synthetic spectrum discussed in the text with $T_{\text {eff }}=7500 \mathrm{~K}$ at $v \sin i=5$ (left) and $150 \mathrm{~km} \mathrm{~s}^{-1}$ (right). Middle panel: same for $T_{\text {eff }}=8000 \mathrm{~K}$. The bottom panel shows the cross-correlation peak in both cases, centred at $+0.01 \mathrm{~km} \mathrm{~s}^{-1}$ for $v \sin i=5 \mathrm{~km} \mathrm{~s}^{-1}$ and at $-12.3 \mathrm{~km} \mathrm{~s}^{-1}$ for $v \sin i=150 \mathrm{~km} \mathrm{~s}^{-1}$ (in the sense: $7500 \mathrm{~K}$ spectrum with respect to $8000 \mathrm{~K}$ spectrum)

(1991) and Fekel (1999) for the 10 non-variable stars common to both studies and obtained $0.3 \pm 0.9 \mathrm{~km} \mathrm{~s}^{-1}$, thus adding firmness to the earlier conclusion. In Sect. 4.4 we confirm the appropriateness of that wavelength region for the stars studied by Fekel, and indicate how the situation changes for higher rotational velocities.

\subsection{A closer look at mismatch}

The following example illustrates the problems we are confronting here. Consider two noise-free synthetic spectra generated with the same rotational velocity $\left(150 \mathrm{~km} \mathrm{~s}^{-1}\right)$, abundances (solar) and surface gravity $(\log g=4.0)$, but with $T_{\text {eff }}=8000 \mathrm{~K}$ (spectral-type $\left.\sim \mathrm{A} 7 \mathrm{~V}\right)$ and $7500 \mathrm{~K}$ (spectral-type $\sim$ A9V), respectively. The spectra were degraded to a resolution of $\sim 0.12 \AA$ per pixel at $4300 \AA$, and we select a wavelength interval 3717.1 $5158.0 \AA$ A. Obviously there should be no Doppler shift between those spectra. Yet if we cross-correlate them, we find that the maximum of the cross-correlation funtion is at $2.2 \mathrm{~km} \mathrm{~s}^{-1}$ instead of zero. If we then take smaller subregions, $4684.9-4724.9 \AA$ and $5045.7-5060.9 \AA$, the mismatch shifts are $0.1 \mathrm{~km} \mathrm{~s}^{-1}$ and $12.3 \mathrm{~km} \mathrm{~s}^{-1}$, respectively. So the mismatch shift is by no means uniquely related to the difference in the atmospheric parameters only. A more detailed inspection shows that its magnitude and even its sign depend in the first place on the wavelength interval used in the cross-correlation, or rather on the behaviour and the importance of the blends therein; any blend which contributes significantly to the cross-correlation function and whose asymmetry is sensitive to $T_{\text {eff }}$ and $\log g$, may cause serious trouble in this context. For the last wavelength region considered above, Fig. 1 illustrates how differences, between object and template spectrum, in the relative strengths of nearby lines are harmless as long as lines are symmetrical and isolated - as is statistically the case at low rotational velocities. However, the fact that the relative strength of the Fe I and $\mathrm{NiI}$ lines shortward of $5052 \AA$ with respect to the CI line just longward of $5052 \AA$ is significantly larger at $7500 \mathrm{~K}$ than it is at $8000 \mathrm{~K}$ places more weight, for higher rotational velocities, on the short-wavelength side of the blend at the lower temperature, causing a (large) displacement of the crosscorrelation maximum.

A limited quantitative analysis of the connection between mismatch, caused by simple but specific differences in blending between object and template spectrum, and the resulting mismatch shift was presented by Verschueren (1991). He conducted cross-correlation experiments with a single, well-sampled, synthetic Gaussian line as template, and an object spectrum consisting of the same line blended with a weaker Gaussian line of the same width. Using the width $\sigma$ of the lines as the unit of length (the set-up is scale-invariant), and varying the distance $d$ between the two line-centres in the object spectrum and the line-strengths of the primary $\left(I_{1}\right)$ and blending $\left(I_{2}\right)$ line, he concluded the following:

- the mismatch shift vanishes at $d=0$ and $d>\sim 6 \sigma$; it reaches a maximum somewhere in the interval $\sigma<$ $d<2 \sigma$;

- for a given $I_{1}$, the mismatch shift is proportional to $I_{2}$; for a given $I_{2} / I_{1}$, it depends only weakly on $I_{1}$;

- using a wavelength interval containing $n$ similar blends (isolated from each other) and $m$ isolated lines similar to the primary line in the blends, then the mismatch shift is approximately proportional to $n /(n+m)$.

Since these results were obtained for an extremely simplified situation, we can only expect to be able to make qualitative comparisons with results from stellar spectra. In Sect. 4.3, we will see that this can be done to some extent when studying the influence of increased rotational broadening. 


\subsection{Proposed methodology}

A straightforward approach to optimize the accuracy of RV measurements might seem to be to hunt down all "badly behaved" blends (i.e. those sensitive to small changes in temperature or gravity) and somehow eliminate them from the cross-correlation process. However, one may then end up with so little "good" material left that random errors become very high; this is especially true for fast rotators, partly because of increased blending and also because their cross-correlation peak positions are more sensitive to noise. The selection process must therefore take into account the effects of noise in the data, and seek a satisfactory compromise between random errors due to noise and systematic errors due to mismatch. Furthermore, eliminating a blend almost inevitably introduces into the data two artificial discontinuities, which may cause a serious systematic error (henceforth referred to as "end effect"). One may therefore be obliged to include wider spans, tolerating some bad blends and sacrificing some good regions.

At this point one could choose an ab initio approach to search for harmful blends by comparing line-lists of all elements contributing to the spectra and deducing, from the way their strengths vary with $T_{\text {eff }}$ and $\log g$, the behaviour of the blends according to a particular broadening law. Instead, we preferred a more phenomenological approach, since it offers directly a link with the magnitude of the induced mismatch shift:

- generate synthetic stellar spectra over a fairly wide wavelength range for fixed (solar) abundances, forming a grid of $T_{\text {eff }}$ and $\log g$ plus a range in $v \sin i$;

- divide the whole wavelength range into a number of intervals subject to the requirements outlined in Sect. 3.4;

- for each interval, calculate the mismatch shifts which occur when cross-correlating it with the corresponding intervals in a set of neighbouring spectra in the $\left(T_{\text {eff }}\right.$, $\log g$ )-grid.

The use of synthetic spectra for this purpose offers a number of advantages: an arbitrarily dense grid in $T_{\text {eff }}$ and $\log g$, perfect control over the parametrization, a large wavelength range, RVs that are exactly zero, and absence of noise. Even though synthetic spectra cannot be expected to be fully realistic in an absolute sense, their use here is justified because we only employ them differentially. Thus we anticipate that the synthetic spectral mismatch caused by a small difference in atmospheric parameters is in fact sufficiently realistic to reproduce the same kind of mismatch shifts one would find between real spectra, thereby providing us with the insight we are seeking. Obviously, any definite conclusions and a fortiori any tentative strategy, emerging from such a study, to reduce the errors must be verified using real spectra.

We shall not include any mismatch arising from abundance differences between object and template spectrum, as the parameter-space would become unmanageable; incidentally, that assumption may be quite appropriate for cross-correlating different stars from the same stellar cluster (e.g. Vrancken et al. 1997). Abundance differences can be included at a later stage when studying individual stellar peculiarities in real spectra, as explained in Sect. 6 . We also shall not consider mismatch arising through differences in rotational velocity because that can be avoided up to almost any accuracy by applying artifical rotational broadening to the template, and because - in the absence of other mismatch and of low-frequency filtering - it produces negligible systematic errors (Verschueren 1991).

\section{Experimental set-up}

\subsection{Synthetic spectra}

Classical LTE line-blanketed atmosphere structures, having a solar composition and $v_{\text {turb }}=2 \mathrm{~km} \mathrm{~s}^{-1}$, were taken from the grid of Kurucz (1979, 1993). Corresponding LTE spectra were computed with the programme SYNSPEC (Hubeny et al. 1994), which takes Kurucz line lists (Kurucz 1988; Kurucz \& Bell 1995) as input. Metalline broadening was computed from classical expressions given by Kurucz (1979), while tables given by Vidal et al. (1973), Barnard et al. (1974) and Shamey (1969) were used for $\mathrm{H}$ and He I.

The spectra were computed from the Balmer jump to $5200 \AA$ and are sampled with a constant step of $2810^{-6}$ in $\ln \lambda$ (corresponding to a resolution of $\sim 0.12 \AA$ per pixel at $4300 \AA)$. This step-size provides that all apparent features in spectra broadened by $\sim 15 \mathrm{~km} \mathrm{~s}^{-1}$ or more are well sampled. Since line blending is the cause of the errors we are studying, our results will not be strictly applicable quantitatively to spectra in which the same blends are not well sampled owing to inferior resolution (depending on $v \sin i$ ). This is in principle the case for our own synthetic spectra with $v \sin i=5 \mathrm{~km} \mathrm{~s}^{-1}$ : the mismatch shifts we compute from them will be somewhat larger than could ideally be achieved with greater resolution.

Atmospheric parameters were selected in the intervals $T_{\text {eff }}=7000-10000 \mathrm{~K}$ in steps of $250 \mathrm{~K}$, and $\log g=$ $3.8-4.2$ in steps of 0.2 , corresponding roughly to the range early $\mathrm{F}$ - late B sub-giants, dwarfs, and ZAMS stars. The spectra were rotationally broadened by $v \sin i=5,50,100$, 150,200 and $300 \mathrm{~km} \mathrm{~s}^{-1}$. The largest rotational velocity was included only for a qualitative comparison with $v \sin i$ $=200 \mathrm{~km} \mathrm{~s}^{-1}$, since classical rotational broadening becomes a poor approximation near the break-up velocity of a star (see e.g. Collins \& Truax 1995). All spectra were normalized to a pseudo-continuum level by a smooth function fitted to the flux maxima between the Balmer lines of the spectra in which $v \sin i=5 \mathrm{~km} \mathrm{~s}^{-1}$. 


\subsection{Spectrum mismatch cases}

This phenomenological study has to simulate the spectral-type mismatch that is likely to occur between two main-sequence A-type stars. When comparing an observed, non-abnormal spectrum with the templates from a synthetic grid, the uncertainty in matching is probably not more than one sub-class in $T_{\text {eff }}(\sim 250 \mathrm{~K})$ and about half a class in $\log g(0.1 \mathrm{dex})$. Thus, if we compute relative RVs between different observed stars whose classifications are each uncertain by that amount, the spectral-type mismatch may be doubled. Also, when establishing the relative RVs of candidate standard stars in a grid whose separations are one sub-class in temperature and half a class in gravity, we must attain high accuracy when bridging at least two such separations in order to ensure zero-point consistency throughout the whole system. For this study, we therefore investigate spectral-type mismatch produced by differences of up to two sub-classes in temperature $\left( \pm 500 \mathrm{~K}\right.$ in $\left.T_{\text {eff }}\right)$ and up to one class in gravity $( \pm 0.2$ dex in $\log g$ ).

In practice, that concept is translated into a "mismatch grid" of 14 spectra, centred on a spectrum with given $T_{\text {eff }}, \log g$ and $v \sin i$ and differing from it by one and two sub-classes in $T_{\text {eff }}$ and by one class in $\log g$ in either direction, respectively. Those 14 spectra represent 14 possible "mismatch cases" to be considered for the central spectrum. Note that the difference of 0.2 dex we consider in $\log g$ was not subdivided further since mismatch shifts arising from this difference are already much smaller in general than shifts arising from the selected temperature mismatch. For the reasons given in Sect. 2.3, we disallowed the possibility of mismatch in $v \sin i$.

\subsection{Mismatch shifts and mismatch errors}

In order to study how spectral-type mismatch errors behave throughout the A-type main-sequence, we consider a "main grid" of 30 spectra having $T_{\text {eff }}=7500,8000,8500$, 9000 and $9500 \mathrm{~K}, \log g=4.0$, and $v \sin i=5,50,100$, 150,200 and $300 \mathrm{~km} \mathrm{~s}^{-1}$. These spectra may be regarded as templates. Each was then cross-correlated with the 14 spectra of its mismatch grid defined in Sect. 3.2; the latter spectra may be regarded as 14 object spectra which, because of our assumed classification uncertainties, may all be cross-correlated with the same template. All crosscorrelations are carried out using the different spectral regions appropriate for each of the main-grid spectra (see Sect. 3.4). The result of each cross-correlation is a mismatch shift, defined as the difference in velocity caused by the mismatch between both spectra in the spectral region considered.

Next, for each of the 30 main-grid spectra, and for each spectral region, we define the expected "mismatch error"
$E_{\mathrm{RV}}$ as the maximum of the absolute values of the mismatch shifts derived for the 14 different mismatch cases. Since in practice one does not know which of the 14 cases occurs when cross-correlating two observed spectra, and since mismatch shifts are purely systematic in nature, the only useful parameter for characterizing the quality of a spectral region is the maximum value of all possible errors.

In summary, we have selected 30 main-grid spectra representing the A-type main-sequence stars at different rotational velocities; for each one we stipulated 14 probable cases of spectral-type mismatch and computed for each one the mismatch shift in velocity for a few tens of spectral regions. We defined, for each of the main-grid spectra, the mismatch error of each spectral region to be the highest of those shifts.

Cross-correlations were performed with the CORSPEC package, updated from Verschueren (1991). In order to minimize end effects, the flux in each spectral region was first rescaled to the average level between its two end points; an end-masking of 5 pixels length was then additionally applied. Mismatch shifts were computed by fitting a parabola through the highest 3 pixels of the crosscorrelation peak, taking into account the discretization correction of David \& Verschueren (1995). The formal random error for each cross-correlation was also computed using the theoretical expression given by Verschueren \& David (1999) and adopting reference $S / N$ values of 50 , 100 and 200, respectively, in the continuum of the object spectrum and infinity for the template. The $S / N$ in the continuum is assumed to be independent of wavelength, although in practice it depends on the intrinsic energy distribution of the spectrum and on interstellar, telluric and instrumental characteristics.

\subsection{Spectral regions}

Since we want to examine the behaviour of different parts of the spectra, we have to define a set intervals, which we call "spectral regions", for the main-grid of 30 spectra defined in Sect. 3.3. Owing to the large differences in character between the different spectra, it proved impossible to select a unique set of spectral regions that was equally suitable in all cases. Nevertheless, we tried to define them as consistently and as homogeneously as possible for all the spectra considered, making neither their length too large that individuality was lost, nor so small that we ran the risk of low information content and important end effects.

First, in all 30 main-grid spectra, we searched in an automated way for "continuum windows", defined here as spectral intervals in which the normalised flux is greater than 0.99 over a minimum span of 10 pixels for all nearby spectra with which the given spectrum will have to be cross-correlated (i.e. the set of 14 mismatch cases to be considered; see Sect. 3.2). A length of 10 pixels provides 
Table 1. Master list of central wavelengths of continuum windows. Those windows bracketing a $\mathrm{H}$ line are indicated by that line in the last column. Continuum windows 01 and 02 bracket the Balmer series H14-H8. The start and end wavelength of all spectral regions for all spectra are chosen from this list. Throughout this paper, spectral regions will be noted by the combination of their numbers as appearing in the first column (e.g. region No. 08-12 runs from $4212.5-4405.5 \AA$ )

\begin{tabular}{cccc|cccc}
\hline No. & $\lambda(\AA)$ & $\ln (\lambda)$ & & No. & $\lambda(\AA)$ & $\ln (\lambda)$ & \\
\hline 01 & 3717.1 & 8.2207 & & 19 & 4608.7 & 8.4357 & \\
02 & 3924.5 & 8.2750 & $\mathrm{H} \epsilon$ & 20 & 4623.9 & 8.4390 & \\
03 & 4019.8 & 8.2990 & $\mathrm{H} \epsilon$ & 21 & 4641.5 & 8.4428 & \\
04 & 4039.2 & 8.3038 & & 22 & 4661.1 & 8.4470 & \\
05 & 4050.1 & 8.3065 & $\mathrm{H} \delta$ & 23 & 4688.2 & 8.4528 & \\
06 & 4159.7 & 8.3332 & $\mathrm{H} \delta$ & 24 & 4725.8 & 8.4608 & \\
07 & 4193.1 & 8.3412 & & 25 & 4750.5 & 8.4660 & \\
08 & 4212.5 & 8.3458 & & 26 & 4778.1 & 8.4718 & $\mathrm{H} \beta$ \\
09 & 4231.4 & 8.3503 & & 27 & 4944.3 & 8.5060 & $\mathrm{H} \beta$ \\
10 & 4257.3 & 8.3564 & & 28 & 4974.1 & 8.5120 & \\
11 & 4266.3 & 8.3585 & $\mathrm{H} \gamma$ & 29 & 4995.0 & 8.5162 & \\
12 & 4405.5 & 8.3906 & $\mathrm{H} \gamma$ & 30 & 5026.1 & 8.5224 & \\
13 & 4439.1 & 8.3982 & & 31 & 5045.7 & 8.5263 & \\
14 & 4478.3 & 8.4070 & & 32 & 5061.9 & 8.5295 & \\
15 & 4486.4 & 8.4088 & & 33 & 5092.4 & 8.5355 & \\
16 & 4511.6 & 8.4144 & & 34 & 5115.9 & 8.5401 & \\
17 & 4537.8 & 8.4202 & & 35 & 5158.0 & 8.5483 & \\
18 & 4569.2 & 8.4271 & & 36 & 5177.6 & 8.5521 & \\
\hline
\end{tabular}

enough scope to ensure sufficiently flat ends in the event of even somewhat larger mismatches, or to accommodate actual Doppler shifts between observed spectra. For higher rotational velocities, the value of 0.99 had to be relaxed to 0.98 or 0.97 in order to find a reasonable number of continuum windows. For the late A-type spectra, no continuum windows shortward of $\mathrm{H} \delta$ were found because of the increasing metal-line density in the Balmer-line wings; we decided simply to use the ones suitable for the other spectra in this wavelength region, and verified that resulting end effects were negligible in these relatively wide $\mathrm{H}$-line regions.

From these data, a "master list" of 36 continuum windows was generated which provided a satisfactory representation of all 5 main-grid spectra at $v \sin i=5 \mathrm{~km} \mathrm{~s}^{-1}$. Table 1 lists their central wavelengths. At higher rotational velocities, some of those 36 windows cease to exist above a certain value of $v \sin i$ because of line broadening, the more so for greater line-densities (i.e. lower temperatures). We therefore created sub-lists of continuum windows for different values of $v \sin i$ and $T_{\text {eff }}$, keeping the number as high as possible and the selection as homogeneous as possible across all temperatures and rotational velocities. In that way we retained 13 continuum windows at $v \sin i=300 \mathrm{~km} \mathrm{~s}^{-1}$ for $T_{\text {eff }}=7500 \mathrm{~K}$, and 23 for $T_{\text {eff }}=9500 \mathrm{~K}$.

"Spectral regions" were then defined simply as the intervals between adjacent continuum windows taken from the relevant sub-list, the whole set thus covering the entire spectrum synthesized in each case. A final iteration of the continuum windows was then carried out in order to ensure robustness against end-effects: we checked that endmasking and the actual location (up to \pm 5 pixels) of the extremities of the spectral regions was not critical for the cross-correlations. These tests resulted in the removal of only a few continuum windows from some sub-lists defined for $v \sin i \geq 100 \mathrm{~km} \mathrm{~s}^{-1}$. Owing to the constraints we imposed on the continuum windows, some of the small spectral regions merge into larger ones as $v \sin i$ increases and $T_{\text {eff }}$ decreases. The final lists of spectral regions include, for all spectra, 5 "H-line regions": the H14-H8 Balmer line region, one with $\mathrm{H} \epsilon$ and CaII $\mathrm{K}$, and those with $\mathrm{H} \delta, \mathrm{H} \gamma$ and $\mathrm{H} \beta$ - each of course blended with other lines. Regions without $\mathrm{H}$ lines (the "metal-line regions") have an average width of $\sim 25 \AA$ if $v \sin i=5 \mathrm{~km} \mathrm{~s}^{-1}$ and $\sim 40 \AA$ if $v \sin i$ $=300 \mathrm{kms}^{-1}$.

\section{Mismatch errors of individual spectral regions}

In this section, we present the expected mismatch errors $E_{\mathrm{RV}}$ of the individual spectral regions. We recall that each is defined as the maximum mismatch shift (in absolute value) out of the 14 different mismatch cases (two steps of $\pm 250 \mathrm{~K}$ in $T_{\text {eff }}$ and one step of \pm 0.2 dex in $\log g$; see Sect. 3.2). We discuss these results for the 30 main-grid spectra considered, i.e. for the 5 values of temperature, 1 of surface gravity, and 6 of rotational velocity, representing the rotating A-type main-sequence stars (see Sect. 3.3). First, in Sect. 4.1, we look in more detail at the individual mismatch shifts arising from the 14 different mismatch cases.

\subsection{Mismatch cases and shifts}

The step in $\left(T_{\text {eff }}, \log g\right)$ of the mismatch grid as chosen in Sect. 3.2 needs to be sufficiently small to sample properly the mismatch error $E_{\mathrm{RV}}$ as a function of $\Delta T_{\text {eff }}$ and $\Delta \log g$. We are satisfied that the latter requirement has in fact been met because a simple four-parameter model $\left(A \Delta T_{\text {eff }}+B \Delta T_{\text {eff }}^{2}+C \Delta \log g+D \Delta T_{\text {eff }} \Delta \log g\right)$ fits the 14 mismatch shifts fairly well for most spectral regions in all main-grid spectra. We also found that the maximum mismatch shift, i.e. the mismatch error $E_{\mathrm{RV}}$, for $\Delta T_{\text {eff }}=500 \mathrm{~K}$ (i.e. for 14 mismatch cases) is twice that for $\Delta T_{\text {eff }}=250 \mathrm{~K}$ (i.e. for 8 mismatch cases), with an rms deviation less than 0.5 for all 30 main-grid spectra and for all spectral regions.

As pointed out in Sect. 1, if spectra have a high linedensity one tends to assume that, for a given mismatch case, the mismatch shifts caused by individual blends in a given spectrum are like random numbers distributed symmetrically around zero. Visual inspection of our mismatch 


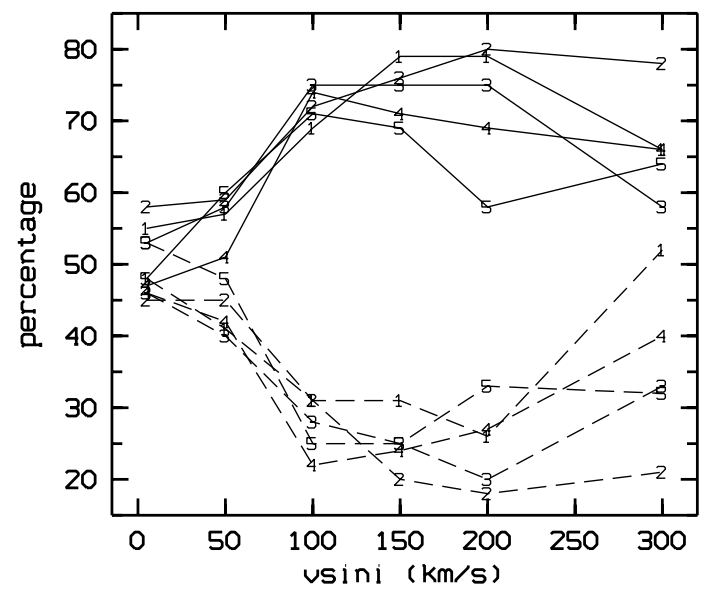

Fig. 2. Percentage of individual metal-line regions with a positive mismatch shift for those mismatch cases with $\Delta T_{\text {eff }}>0$ (full line), and for those with $\Delta T_{\text {eff }}<0$ (dashed line). All mismatch cases in $\log g$ are taken together. Results are shown for each of the 30 spectra separately with the numerical labels referring to their $T_{\text {eff }}(1=7500 \mathrm{~K}, 2=8000 \mathrm{~K}, 3=8500 \mathrm{~K}$, $4=9000 \mathrm{~K}, 5=9500 \mathrm{~K})$. If $\Delta T_{\text {eff }}>0(<0)$, the mismatch shift is that of a hotter (cooler) object with respect to the template whose $T_{\text {eff }}$ is referred to by their labels

shifts (see e.g. Fig. 2) suggests that, on the contrary they have a preferential sign in different spectral regions for many of the spectra and mismatch cases. Although the wavelength regions defined in Sect. 3.4 do not isolate individual blends, those without hydrogen lines are sufficiently narrow and numerous to allow a limited verification of the assumption of randomness of the sign. For each of the 420 samples of mismatch shifts taken from all metal-line regions (for 14 mismatch cases in 30 spectra), we tested the hypothesis that the sign of the shifts in the sample is random. The results can be summarized as follows:

- at the $5 \%$ significance level the hypothesis cannot be rejected for any of the 30 spectra if the mismatch is caused only by differences in $\log g$ and not in $T_{\text {eff }}$;

- likewise it cannot be rejected for any mismatch case if the template spectrum has $v \sin i=5$ or $50 \mathrm{~km} \mathrm{~s}^{-1}$;

- however, for all temperatures there are one or two $v \sin i$ values and several mismatch cases for which the hypothesis must indeed be rejected at the $5 \%$ level, with a slight preponderance of cases with $\Delta T_{\text {eff }}>0$, $\Delta \log g=0$ and $T_{\text {eff }}>7500 \mathrm{~K}$; the total number of these cases is 110 , and in 42 out of them the hypothesis must be rejected even at the $1 \%$ level so it is quite unlikely that they could be ascribed to coincidence.

A characteristic of these trends is illustrated in Fig. 2, where a strong correlation appears between the sign of the temperature mismatch and the sign of the corresponding mismatch shift, for most metal-line regions and for rotational velocities above $\sim 50 \mathrm{kms}^{-1}$. The correlation exists for all temperatures, although the scatter increases towards the highest $v \sin i$ because of the smaller number of regions defined. The reason for this common behaviour of most spectral regions has yet to be investigated by examining the origin of each shift. Positional relations between the lines of multiplets contributing to temperaturedependent blends in different regions presumably play a role. The important consequence is that, at least for faster rotators, a majority of the metal-line blends may cause a mismatch shift in the same sense so that one cannot be certain of reducing systematic errors simply by including more such blends in a cross-correlation. This will strongly influence the extent to which it is useful to combine individual spectral regions as is discussed in Sect. 5.1.

\subsection{Distribution}

For each of the 30 main-grid spectra we plotted the distribution of mismatch errors $E_{\mathrm{RV}}$ from the different spectral regions. A Kolmogorov-Smirnov test shows that, for a given rotational velocity, the hypothesis that the distributions for the 5 different temperatures are identical cannot be rejected, although the relatively small number of regions must influence that result. We can therefore combine the results for all $T_{\text {eff }}$; Fig. 3 shows the distributions for the 6 values of $v \sin i$. It is immediately apparent that a large range in $E_{\mathrm{RV}}$ exists among the different regions at any given $v \sin i$ : the $10 \%$ highest errors are on average about a factor 20 larger than the $10 \%$ smallest ones.

The most important characteristic of these distributions is the strong and consistent increase in the errors with $v \sin i$. In Sect. 4.3 we will quantify that dependence in more detail. Although the distributions of all $E_{\mathrm{RV}}$ for different temperatures are indistinguishable, the mismatch error of each individual region may vary significantly with $T_{\text {eff }}$, as discussed in Sect. 4.4. The smallest mismatch errors from the $\mathrm{H}$-line regions are of very much the same magnitude as the errors of the best metal-line regions, while at the same time $\mathrm{H}$-line regions are never found among those regions that give rise to the highest errors. A more detailed discussion of the $\mathrm{H}$-line regions is given in Sect. 4.5.

\subsection{Dependence on rotation}

As Fig. 3 shows, the global distribution of mismatch errors scales approximately linearly with $v \sin i$. In order to investigate this dependence more quantitatively for individual spectral regions, those regions were selected that contain exactly the same interval in wavelength for all values of $v \sin i$. This was repeated for the 5 values of $T_{\text {eff }}$ separately in order to detect a possible further dependence on temperature.

A linear relation between the mismatch error from a given spectral region and $v \sin i$, assuming the error is zero 

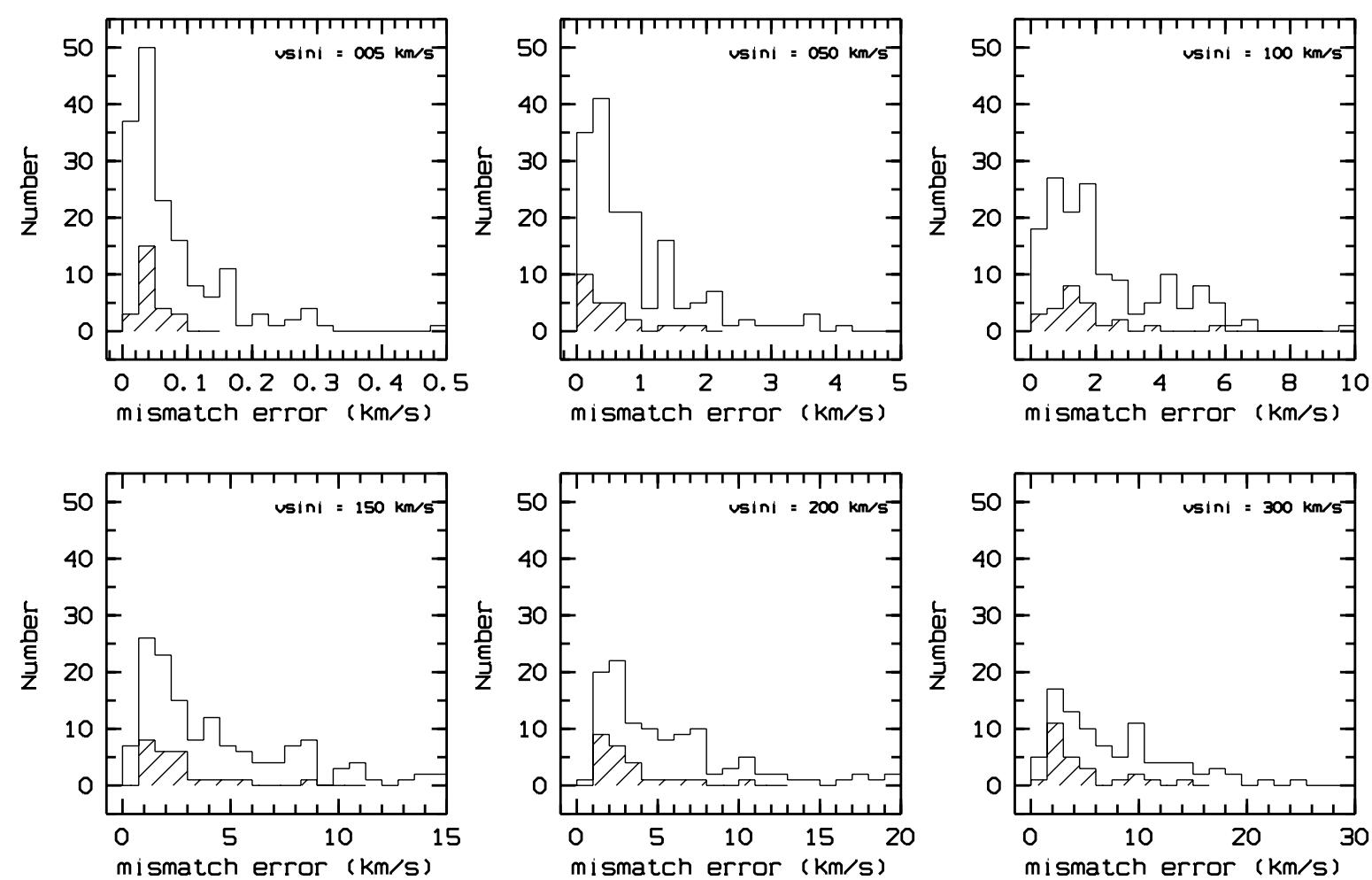

Fig. 3. Distribution of the mismatch errors $E_{\mathrm{RV}}$ from all spectral regions. For each value of $v \sin i$, the results from the 5 values of $T_{\text {eff }}$ are combined. Results for H-line regions are shown hashed

if $v \sin i=0$, can conveniently be expressed on a logarithmic scale:

$\log \left(\frac{E_{\mathrm{RV}}}{E_{\mathrm{RV}}^{(5)}}\right)=\log \left(\frac{v \sin i}{5}\right)$

with $E_{\mathrm{RV}}^{(5)}$ the mismatch error at $v \sin i=5 \mathrm{~km} \mathrm{~s}^{-1}$ to which the errors for other $v \sin i$ are normalised. Figure 4 shows the mismatch error for $T_{\text {eff }}=8000 \mathrm{~K}$ as a function of $v \sin i$ for all 8 selected regions; it also depicts Eq. (1). The double logarithmic scale has the advantage that a linear dependence of the mismatch error on $v \sin i$ between any two $v \sin i$ values is translated into a connecting line with a gradient of unity, i.e. parallel to Eq. (1).

As the plots in Fig. 4 are not significantly different for different temperatures, we can draw the following conclusions from all of them:

- $E_{\mathrm{RV}}$ increases monotonically with $v \sin i$ in almost all spectral regions, partly because in a majority of the wavelength regions all individual mismatch shifts actually increase monotonically, and partly because $E_{\mathrm{RV}}$, by its very definition, is likely to keep on growing even if some of the individual shifts do not. The behaviour of the individual mismatch shifts themselves (monotonic or not) can often be explained qualitatively in terms of the results of the simple two-line study by Verschueren (1991) (see Sect. 2.2), bearing in mind that the distance between spectral lines in units of their width decreases as rotational broadening increases. In particular, in some spectral regions where the mismatch error exhibits a maximum as a function of $v \sin i$, one finds that the condition for a maximum is indeed reached and passed at some $v \sin i<300 \mathrm{~km} \mathrm{~s}^{-1}$ and that all individual mismatch shifts decrease in absolute magnitude.

- Almost all curves have at least one part where the increase is stronger than linear and at least one part where it is less strong than linear. Furthermore, for any step in $v \sin i$, different spectral regions exhibit a large range in slope around the linear value. Although rotational broadening increases $E_{\mathrm{RV}}$ on average, the details of the blending in each individual region determine the final outcome to a large extent.

- The $v \sin i$-dependence of the mismatch errors arising from $\mathrm{H}$-line regions does not seem any different from that arising from the metal-line regions. The reason is that mismatch errors in $\mathrm{H}$-line regions are caused solely by the blending metal lines (with some exception occurring in the Balmer line region; see Sect. 4.5).

\subsection{Dependence on temperature}

For a given $v \sin i$, the mismatch error $E_{\mathrm{RV}}$ for any given spectral region may vary considerably with the 


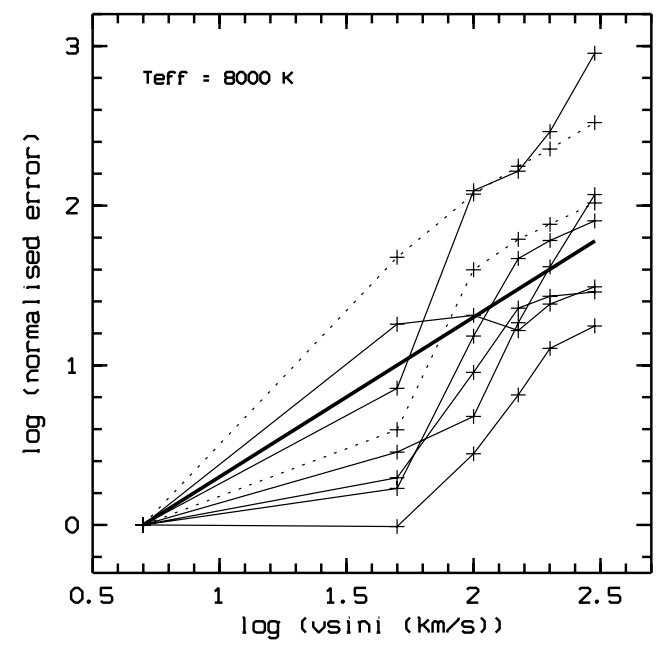

Fig. 4. Normalised mismatch error $E_{\mathrm{RV}}$ as a function of $v \sin i$, plotted on a double logarithmic scale. Different curves represent all spectral regions that contain exactly the same interval in wavelength over the full range of $v \sin i$ for spectra with $T_{\text {eff }}=8000 \mathrm{~K}$. Dotted lines represent H-line regions. The thick line depicts Eq. (1), its slope representing a linear scaling of the error between any two values of $v \sin i$

temperature parameter $T_{\text {eff }}$ of the spectrum. This is shown for $v \sin i=50 \mathrm{~km} \mathrm{~s}^{-1}$ in Fig. 5. The following conclusions can be drawn:

- The mismatch errors of the majority of the spectral regions vary monotononically with temperature, although the sign and the range of the variations varies substantially from one region to the next. Again, the details of the blending in each spectral region are the crucial factor;

- Spectral regions with a relatively weak dependence on temperature also have a relatively small average $E_{\mathrm{RV}}$. Average errors for these "best" regions are $~ 0.04,0.4$, $1,1.5,2$ and $3 \mathrm{~km} \mathrm{~s}^{-1}$ for $v \sin i=5,50,100,150,200$ and $300 \mathrm{~km} \mathrm{~s}^{-1}$, respectively. Note that these "best" regions are largely different ones for different $v \sin i$; this seriously hampers the selection of regions suitable for all spectra (see Sect. 5);

- Some spectral regions have mismatch errors that vary up to a factor of 10 across the temperature range considered. Such large variations, however, do not occur among H-line regions.

Figure 5 supports a comparison with the work of Fekel (1985, 1999), who has derived accurate RVs for late Band A-type stars that have $v \sin i<50 \mathrm{~km} \mathrm{~s}^{-1}$. Fekel uses the $\mathrm{Mg}$ II line at $4481 \AA$ (situated in our region No. 1415) and the 5 Fe II and Ti II lines between $4500-4525 \AA$ (situated at the end of region No. 15-16 and at the beginning of No. 16-17). Our error in region No. 14-15 (Mg II) remains small for $v \sin i=5 \mathrm{~km} \mathrm{~s}^{-1}$ but it increases drastically with decreasing temperature for $v \sin i=50 \mathrm{~km} \mathrm{~s}^{-1}$ (see Fig. 5). For that value of the rotation the region is

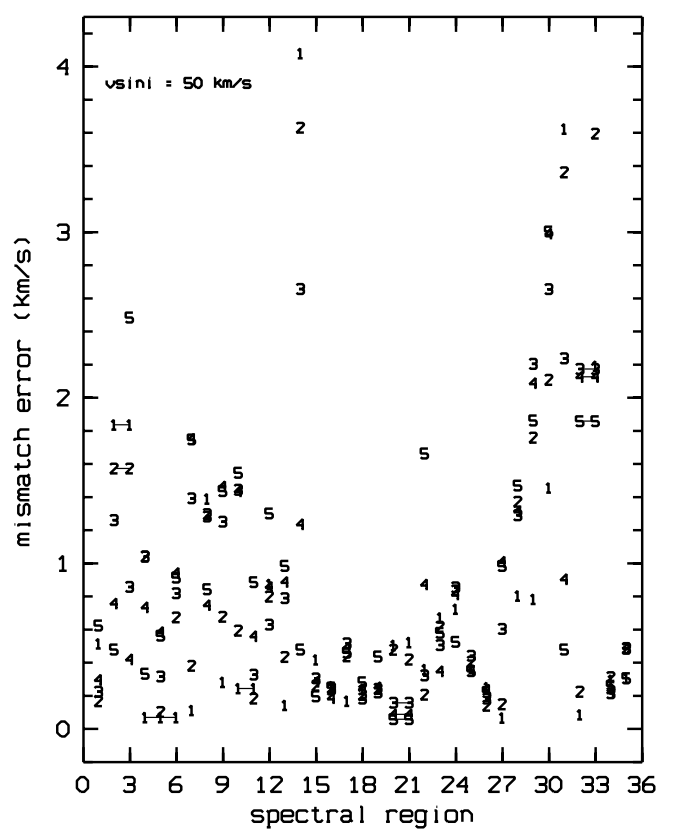

Fig. 5. Mismatch error $E_{\mathrm{RV}}$ as a function of the different spectral regions for 5 different temperatures: $1=7500 \mathrm{~K}$, $2=8000 \mathrm{~K}, 3=8500 \mathrm{~K}, 4=9000 \mathrm{~K}$ and $5=9500 \mathrm{~K}$. The rotational velocity is $50 \mathrm{~km} \mathrm{~s}^{-1}$ in all cases. Spectral regions are indicated by the number of the continuum window corresponding to their starting wavelength (see Table 1). Horizontal full lines indicate the length of a region if it is larger than the distance between two consecutive continuum windows in Table 1

still reliable for early-A spectra, but yields unacceptably high errors for mid- and late-A ones owing to blending by lines which have a strong temperature dependence. Incidentally, the mid and late A-type stars in Fekel's study all have $v \sin i<15 \mathrm{~km} \mathrm{~s}^{-1}$ (Fekel 1999, private communication) which helps to explain the relatively small errors he finds for those stars using the Mg II line. Also for $v \sin i$ $>50 \mathrm{~km} \mathrm{~s}^{-1}$, we find that the Mg II line yields relatively small errors for early A-type spectra only.

The other two regions which contain lines used by Fekel are in fact among the very best, producing mismatch errors between $0.02-0.04 \mathrm{~km} \mathrm{~s}^{-1}$ for $v \sin i=5 \mathrm{~km} \mathrm{~s}^{-1}$ and between $0.2-0.4 \mathrm{~km} \mathrm{~s}^{-1}$ for $v \sin i=50 \mathrm{~km} \mathrm{~s}^{-1}$ (depending on temperature). However, since the maximum mismatch in temperature we have considered spans only 2 temperature sub-classes in either direction, our results indicate that allowing a considerably larger mismatch, such as Fekel $(1985,1999)$ employs, is not without risk for $v \sin i$ approaching $50 \mathrm{~km} \mathrm{~s}^{-1}$. For $v \sin i>50 \mathrm{~km} \mathrm{~s}^{-1}$, our region No. 15-16 remains among the best despite the blending which occurs shortward of Fekel's lines. At that rotational velocity, region No. 16-17 behaves well only in early Atype spectra, and produces relatively high mismatch errors for cooler spectra owing to a complex blend which affects most of Fekel's lines. 


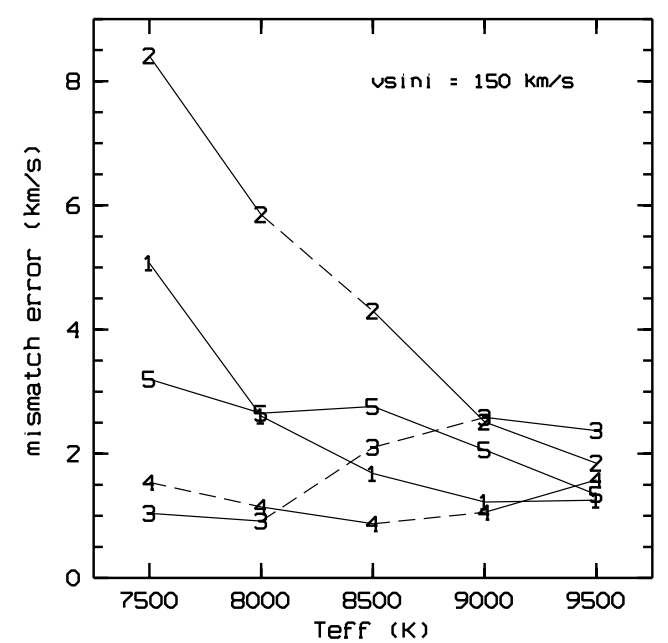

Fig. 6. Mismatch errors $E_{\mathrm{RV}}$ of the 5 H-line regions as a function of spectral temperature for a rotational velocity of $150 \mathrm{~km} \mathrm{~s}^{-1}$. The regions are: $1=\mathrm{H} 14-\mathrm{H} 8$ (Balmer region), $2=\mathrm{H} \epsilon, 3=\mathrm{H} \delta, 4=\mathrm{H} \gamma, 5=\mathrm{H} \beta$. Dashed lines indicate a change in length of the actual wavelength interval, which occurs when a small adjacent metal-line region merges with the H-line region (see Sect. 3.4)

\subsection{A closer look at H-line regions}

Owing to the much larger widths and strengths of the Hydrogen lines compared to the metal lines in these earlytype spectra, spectral regions that contain $\mathrm{H}$ lines require separate investigation. It is important to realize at the outset that the mismatch errors of the 4 regions that contain just one $\mathrm{H}$ line differ from zero only because of the $T_{\text {eff- }}$ and $\log g$-dependent blending with metal lines; the $\mathrm{H}$ line itself is always isolated and symmetric. On the one hand, mismatch errors arising from those $4 \mathrm{H}$-line regions are expected to be smaller on average than those arising from regions with only metal lines because the strong, broad $\mathrm{H}$ line dampens extraneous contributions while not itself contributing to the mismatch; smaller errors for H-line regions are expected also because they have a much larger wavelength span than metal-line regions and because some degree of cancelling of errors always occurs (see Sect. 5.1). On the other hand, some differences in the characteristics of the metal lines (e.g. a difference in strength of an isolated line) will yield (larger) mismatch shifts if those lines occur on the inclined wings of a $\mathrm{H}$-line profile; in addition, differences in spectrum rectification may be an important source of mismatch for these regions. The nett effect cannot easily be predicted, though the distributions given in Sect. 4.2 already showed that mismatch errors from $\mathrm{H}$ line regions lie well within the range found for metal-line regions.

Figure 6 shows the mismatch errors $E_{\mathrm{RV}}$ of the $5 \mathrm{H}$-line regions for the 5 temperatures considered, and for $v \sin i$ $=150 \mathrm{~km} \mathrm{~s}^{-1}$. From this and similar plots for different $v \sin i$ we conclude:
- Mismatch errors arising from the regions containing $\mathrm{H} \delta, \mathrm{H} \gamma$ and $\mathrm{H} \beta$ (labelled 3, 4 and 5) are relatively small; they show no common dependence on temperature for any $v \sin i$, and in that sense they behave similarly to the metal-line regions. Since it is the detailed content of the metal lines in the $\mathrm{H}$-line regions that determines the mismatch shifts, the addition of adjacent small wavelength regions to a $\mathrm{H}$-line region (see the dashed lines) can have a non-negligible effect;

- For $v \sin i<50 \mathrm{~km} \mathrm{~s}^{-1}$, the situation for region 2 containing $\mathrm{H} \epsilon$ is similar to that of the 3 regions discussed above. For larger rotational velocities, however, mismatch is dominated by the blending with the $\mathrm{Ca}$ II H line, though in mid- or late-A- spectra the $\mathrm{K}$ line also blends with the far wing of $\mathrm{H} \epsilon$; both Ca II lines vary markedly with temperature, growing from a very weak line at late B to strong and broad lines at early F. The resulting mismatch errors show a very strong temperature dependence and are relatively high for mid- and late-A spectra. This particular type of blending occurs only in the $\mathrm{H} \epsilon$ spectral region;

- For small $v \sin i$, the situation for region 1 containing the Balmer-series limit ( $\mathrm{H} 14-\mathrm{H} 8)$ is again similar to that of the other H-line regions. For larger rotational velocities, the errors remain small for early- and mid-A spectra but become relatively large for late-A ones. We suggest the following tentative explanation. At larger $v \sin i$ the mismatch is dominated by the seven mutually blended $\mathrm{H}$ lines; those lines become broader with increasing temperature, reaching a maximum at early A. Their blending therefore alters substantially with temperature for the late A-type spectra, while reaching a more stable, almost temperature-independent, configuration for the early A-type spectra. If that mechanism is indeed the dominant one, and if other factors remained equal, we might expect mismatch errors for that spectral region to increase again for B-type stars, where the $\mathrm{H}$ lines become more narrow with increasing temperature.

\section{Selection and combination of spectral regions}

In this section we experiment numerically with several criteria for excluding the badly-behaved spectral regions in order to find out, from a phenomenological basis, to what extent mismatch shifts can be reduced while at the same time keeping the random error contained. An optimal selection strategy will depend on the systematic error that can be tolerated and on the random error $(S / N)$ that is achievable. In this paper, we assume that the $S / N$ is not in principle the limiting factor. We can therefore concentrate on selection strategies that minimize the systematic mismatch errors in an absolute sense, and compute the $S / N$ necessary to keep the random errors below those systematic ones. 
We consider the set of pure metal-line regions separately from the set of 5 regions containing $\mathrm{H}$ lines. The latter regions have much wider wavelength spans and the two sets have different sensitivities to rotational broadening. In addition, the particular non-random behaviour of the sign of the mismatch shifts (see Sect. 4.1) is only apparent for metal-line regions.

\subsection{Sequential combinations}

We know that, for typical late-type spectra with a high line density and a low rotational velocity, mismatch shifts statistically cancel to a very large extent; systematic errors are therefore small, and random errors can be minimized by combining as many spectral regions as possible. This led us to combine spectral regions in a particular way for each of the 30 main-grid spectra in turn. We commence by selecting that region giving the smallest mismatch error $E_{\mathrm{RV}}$ in each case, and subsequently adding further regions in order of increasing $E_{\mathrm{RV}}$. Each step, which includes one more "individual" region than the previous step, constitutes a "combined region"; the last combined region is thus the combination of either all metal-line regions or all H-line regions. Figure 7a shows the mismatch errors $E_{\mathrm{RV}}$ arising from those combined regions for the case with $T_{\text {eff }}=7500 \mathrm{~K}, v \sin i=50 \mathrm{~km} \mathrm{~s}^{-1}$. Also indicated is the $E_{\mathrm{RV}}$ corresponding to the most recently-added individual region, together with the random error associated with each combined region for three adopted values for the $S / N$ in the object spectrum. In this case of our coolest spectrum with a low rotation, which may be considered as the "late-type analogue", mismatch shifts are effectively cancelled throughout the metal-line regions without increasing the errors above the level for the best individual region. For the H-line regions, however, the inclusion of the region containing $\mathrm{H} 14-\mathrm{H} 8$ and the one containing $\mathrm{H} \epsilon$ strongly increases the error, which can be understood from Sect. 4.5.

The example given in Sect. 2.2 has already shown that statistical cancelling of errors fails in the case of higher temperatures or greater rotational velocities. Figure $7 \mathrm{~b}$ (corresponding to $T_{\text {eff }}=9500 \mathrm{~K}, v \sin i=50 \mathrm{~km} \mathrm{~s}^{-1}$ ) and Fig. 7c (corresponding to $T_{\text {eff }}=8500 \mathrm{~K}, v \sin i=$ $200 \mathrm{~km} \mathrm{~s}^{-1}$ ) do indeed exhibit radically different behaviour from Fig. 7a. Owing to the lower line density and (at higher rotational velocities) to the apparently non-random sign of the mismatch shifts of individual spectral regions (see Sect. 4.1), cancelling of errors becomes very inefficient, and the expected mismatch error may substantially increase when more individual spectral regions with successively higher mismatch errors are added. Some (large) errors may still cancel for certain combinations of spectral regions, but the effect is accidental rather than statistical. Unless one is sure that all details of a synthetic spectrum are realistic, one certainly cannot count on the same cancellation occurring for real spectra. Verschueren et al. (1999) investigated, in a particular case, the extent to which mismatch errors may be reduced by searching explicitly for those combinations of spectral regions that show the largest accidental cancelling. For the reasons just explained, the outcome of such an experiment cannot provide the basis for the choice of a suitable set of spectral regions, at least insofar as only synthetic spectra are considered. Our policy for combining spectral regions is therefore to avoid using individual spectral regions with relatively large mismatch errors.

However, since the curves in Figs. 7b,c do not rise monotonically, the practice of avoiding only those individual spectral regions with the largest $E_{\mathrm{RV}}$ does not necessarily reduce the $E_{\mathrm{RV}}$ of the resulting combined region. The only possible robust strategy is thus to use just those spectral regions with very small individual mismatch errors. The cancelling of errors is then reduced to an acceptable level, though we note that some amount of cancelling is always unavoidable, e.g. as occurs internally within each of the individual spectral regions.

For the purpose of later comparisons, it is instructive to investigate the order of magnitude of the mismatch error $E_{\mathrm{RV}}$ which results when this strategy is taken to its extreme, i.e. in each of the 30 main-grid spectra, we use only that individual region which gives the smallest $E_{\mathrm{RV}}$ (regardless of whether it is a metal- or H-line region). For any given $v \sin i$, these $E_{\mathrm{RV}}$ values show some scatter with $T_{\text {eff }}$, but there is no trend. Their maxima over the 5 values of $T_{\text {eff }}$ are therefore a conservative measure of the maximal RV accuracy attainable at each $v \sin i$, at least when one does not want to depend on cancelling of errors. The dotted curve in Fig. 8a shows this accuracy: RV accuracies below $1 \mathrm{~km} \mathrm{~s}^{-1}$ are only possible for rotational velocities below about $150-200 \mathrm{~km} \mathrm{~s}^{-1}$, but even at $v \sin i=$ $300 \mathrm{~km} \mathrm{~s}^{-1}$ those errors are definitely below $2 \mathrm{~km} \mathrm{~s}^{-1}$. Unfortunately, that result has mostly academic value only, for three reasons. First, since very small wavelength regions are used in the metal-line case, the required $S / N$ necessary to obtain a random error of the same order of magnitude as the systematic error is in the region of 1000 and is thus out of reach for most observing programmes at present. Note that this problem is reversed for a H-line region, where systematic errors are commonly larger than random errors even at moderate $S / N$ (as shown by the dotted curves in Figs. 7). Secondly, that result depends entirely on the realism of just a few spectral features in a synthetic spectrum. And thirdly, the very best spectral region is a different one for different spectra; there is no guarantee that the best regions in two adjacent spectra will yield equally small errors at intermediate values of $T_{\text {eff }}$ or $v \sin i$. 

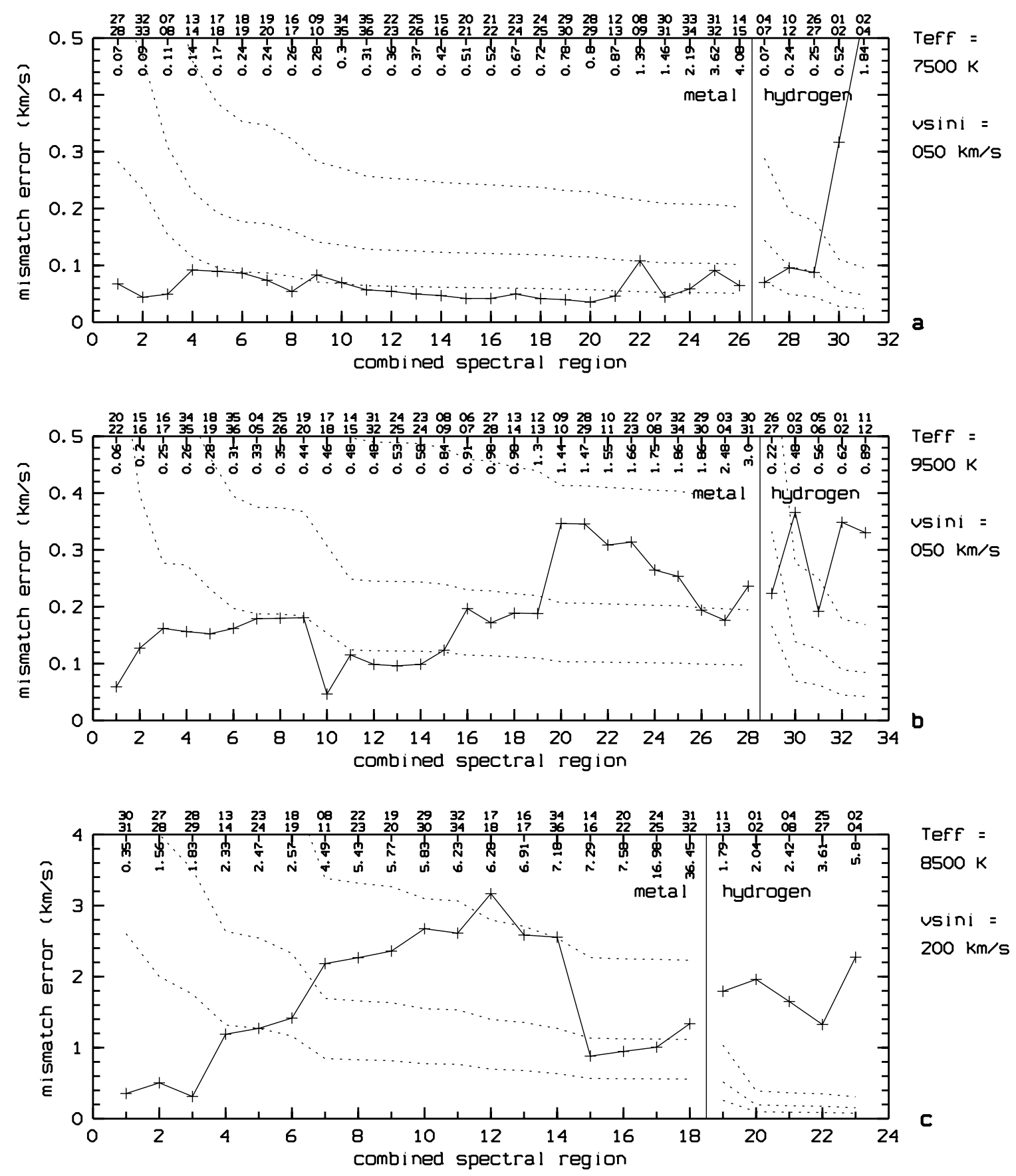

Fig. 7. Crosses and the connecting full line show, for the three spectra indicated on the right, the mismatch error $E_{\mathrm{RV}}$ for the sequentially-combined spectral regions defined in Sect. 5.1. Dotted lines show the random error of each combined region for a $(S / N)_{\mathrm{obj}}=50,100,200$ from top to bottom, respectively, and a $(S / N)_{\text {tem }}=\infty$. Results are shown separately for combinations without, and with, $\mathrm{H}$ lines. Just above the top of the graph is indicated, for each combined spectral region, the number of the last-added individual spectral region (the notation is described in Table 1). The number just below the top of the graph is the $E_{\mathrm{RV}}$ value of the latter expressed in $\mathrm{kms}^{-1}$ 

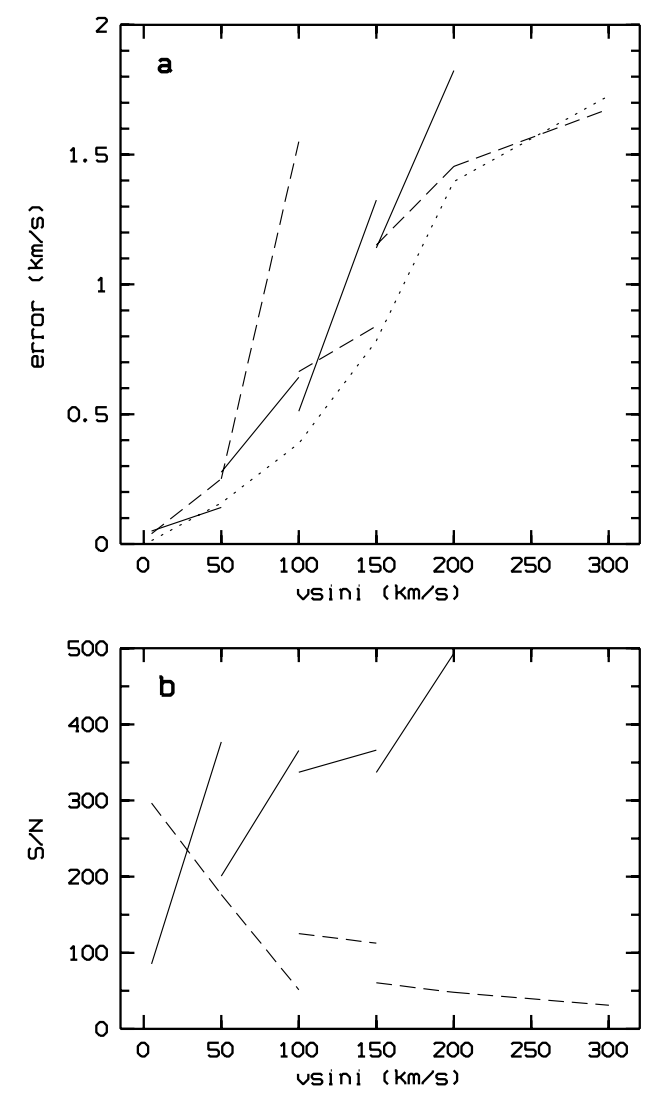

Fig. 8. a) Full lines: Expected RV accuracy attainable for different ranges in $v \sin i$ when using the "uniform" selections of metal-line wavelength regions as explained in Sect. 5.2. The relationship is valid for all A-type main-sequence spectra and for the spectral-type mismatch outlined in Sect. 3.2. For $v \sin i=$ $200-300 \mathrm{~km} \mathrm{~s}^{-1}$, the selection which is tentatively proposed in Table 2 yields errors of about $5 \mathrm{~km} \mathrm{~s}^{-1}$ and is not shown for clarity. Dashed line: Same for the "uniform" selections of H-line regions. Dotted line: Same when using only the best individual spectral region as explained in Sect. 5.1. b) $S / N$ value necessary to obtain a random error equal to the systematic error given by the corresponding type of curve in a)

\subsection{Uniform combinations}

In view of the foregoing discussion we implemented the following strategy for selecting individual spectral regions. First, for each value of $v \sin i$ separately, we selected those individual regions whose derived mismatch error $E_{\mathrm{RV}}$ is small for all $T_{\text {eff }}$ (see e.g. Fig. 5). Because of the need for a sufficient number of selected regions, we selected $E_{\mathrm{RV}}$ cut-off values for the 6 different $v \sin i$ cases of $0.1,0.7,1.5$, $2.5,3$ and $7 \mathrm{~km} \mathrm{~s}^{-1}$, respectively. Even then, the number of suitable regions decreases strongly with $v \sin i$. In order to ensure continuity as a function of $v \sin i$, only those regions common to the selections for two consecutive $v \sin i$ values were then retained for use between those two $v \sin i$ values. This procedure was followed separately for metaland $\mathrm{H}$-line regions but with the same $E_{\mathrm{RV}}$ cut-off values.
Table 2 lists the individual spectral regions finally selected in this way. We note parenthetically that an alternative strategy, of searching at a given $T_{\text {eff }}$ for spectral regions with a small $E_{\mathrm{RV}}$ for all $v \sin i$, was unsuccessful.

Our procedure meets the four requirements following from the discussion in Sect. 5.1. First, accidental cancelling of large errors is avoided because only very good individual regions are used; secondly, enough spectral information is sampled so as to avoid the need for extremely high $S / N$ (see Fig. 8b) and (thirdly), so as not to depend on the details of just a few spectral features in a synthetic spectrum; fourthly, the selection is uniform in $T_{\text {eff }}$ and continuous in $v \sin i$, so the derived errors are valid for the whole of the parameter space.

This selection was then applied to all 30 main-grid spectra and the expected mismatch error $E_{\mathrm{RV}}$ was computed for the standard mismatch defined in Sect. 3.2. At $v \sin i=50,100,150$ and $200 \mathrm{~km} \mathrm{~s}^{-1}$, both relevant selections were applied separately; e.g. at $v \sin i=50 \mathrm{~km} \mathrm{~s}^{-1}$, the selection suitable for $5-50 \mathrm{~km} \mathrm{~s}^{-1}$ and the one suitable for $50-100 \mathrm{~km} \mathrm{~s}^{-1}$ were both computed. For a given $v \sin i$, the same selection was of course applied for the 5 values of $T_{\text {eff }}$. The mismatch errors $E_{\mathrm{RV}}$ were then plotted as a function of $T_{\text {eff }}$ for each value of $v \sin i$ and, again, no significant dependence on temperature was found. Therefore, for each $v \sin i$, the maximum value over the 5 values of $T_{\text {eff }}$ was adopted as a conservative measure of the accuracy attainable with each of the proposed selections. Figure 8a shows this expected accuracy for each of the ranges in rotational velocity considered, and for metal- and $\mathrm{H}$-line regions separately. For $v \sin i$ below about $100 \mathrm{~km} \mathrm{~s}^{-1}$, our selection based on metal-line regions produces consistently smaller errors than that based on $\mathrm{H}$-line regions. For $v \sin i>100 \mathrm{~km} \mathrm{~s}^{-1}$, regions with $\mathrm{H}$ lines are superior. For $v \sin i \leq 150 \mathrm{~km} \mathrm{~s}^{-1}$, errors are consistently smaller than $1 \mathrm{~km} \mathrm{~s}^{-1}\left(\sim 0.05,0.15,0.5\right.$ and $1 \mathrm{~km} \mathrm{~s}^{-1}$ for $v \sin i=5,50$, 100 and $150 \mathrm{~km} \mathrm{~s}^{-1}$, respectively), while for higher rotation errors in the range $1-2 \mathrm{~km} \mathrm{~s}^{-1}$ can be expected. We recall that these error estimates are conservative values in the sense that they are maxima of errors over all temperatures, which in their turn were based on the maximum mismatch shift of all 14 mismatch cases in $T_{\text {eff }}$ and $\log g$ considered (see Sect. 3.2).

Figure 8b shows the $S / N$ necessary for the random error to equal the systematic error of Fig. 8a for each of the proposed selections of spectral regions. For the metal-line selections with $v \sin i \leq 100 \mathrm{~km} \mathrm{~s}^{-1}, S / N$ values roughly between $100-400$ are required, while much smaller values are sufficient if the $\mathrm{H}$-line regions are selected when $v \sin i>100 \mathrm{~km} \mathrm{~s}^{-1}$. The latter stems from the fact that on the one hand the intrinsic cross-correlation power in the H-lines decreases only slightly with rotational velocity, while on the other hand the systematic error increases much more. 
Table 2. Numbers of the individual spectral regions selected for the "uniform" combinations for different ranges of $v$ sin $i$ as discussed in Sect. 5.2. They are suitable for the whole A-type main-sequence. The notation is taken from Table 1. For the H-line regions, the exact definition of the regions may change with temperature and rotation because of the changing availability of continuum windows around the $\mathrm{H}$ lines (see Sect. 3.4)

\begin{tabular}{|c|c|c|c|c|c|c|}
\hline$v \sin i\left(\mathrm{~km} \mathrm{~s}^{-1}\right)$ & \multicolumn{6}{|c|}{ spectral regions } \\
\hline $005-050$ & \multicolumn{6}{|c|}{$15-16,16-17,17-18,18-19,19-20,20-22,23-24,34-35$} \\
\hline $050-100$ & \multicolumn{6}{|c|}{$15-16^{*}, 17-18,18-19,23-24$} \\
\hline $100-150$ & \multicolumn{6}{|c|}{$15-16^{*}, 18-19,23-24,27-28$} \\
\hline $150-200$ & \multicolumn{6}{|c|}{$18-19,23-24,27-28,28-29^{*}$} \\
\hline $200-300$ & \multicolumn{6}{|c|}{$18-19^{*}, 28-29^{*}$} \\
\hline \multirow{3}{*}{$005-050$} & & $7500 \mathrm{~K}$ & $8000 \mathrm{~K}$ & $8500 \mathrm{~K}$ & $9000 \mathrm{~K}$ & $9500 \mathrm{~K}$ \\
\hline & $\mathrm{H} \beta(005)$ & $26-27$ & $26-27$ & $26-27$ & $26-27$ & $26-27$ \\
\hline & $\mathrm{H} \beta(050)$ & $26-27$ & $26-27$ & $26-27$ & $26-27$ & $26-27$ \\
\hline \multirow[t]{2}{*}{$050-100$} & $\mathrm{H} \beta(050)$ & $26-27$ & $26-27$ & $26-27$ & $26-27$ & $26-27$ \\
\hline & $\mathrm{H} \beta(100)$ & $25-27$ & $25-27$ & $25-27$ & $25-27$ & $25-27$ \\
\hline \multirow[t]{2}{*}{$100-150$} & $\mathrm{H} \gamma+\mathrm{H} \beta(100)$ & $09-13,25-27$ & $11-13,25-27$ & $11-13,25-27$ & $11-12,25-27$ & $11-12,25-27$ \\
\hline & $\mathrm{H} \gamma+\mathrm{H} \beta(150)$ & $09-13,25-27$ & $11-13,25-27$ & $11-13,25-27$ & $11-12,25-27$ & $11-12,25-27$ \\
\hline \multirow[t]{2}{*}{$150-200$} & $\mathrm{H} \delta+\mathrm{H} \gamma(150)$ & 04-08, 09-13 & $04-08,11-13$ & $04-06,11-13$ & $05-06,11-12$ & $05-06,11-12$ \\
\hline & $\mathrm{H} \delta+\mathrm{H} \gamma(200)$ & $04-08,08-16$ & $04-08,08-13$ & $04-08,11-13$ & $04-06,11-12$ & $04-06,11-12$ \\
\hline \multirow[t]{2}{*}{$200-300$} & $\mathrm{H} \delta+\mathrm{H} \gamma(200)$ & $04-08,08-16$ & 04-08, 08-13 & $04-08,11-13$ & $04-06,11-12$ & $04-06,11-12$ \\
\hline & $\mathrm{H} \delta+\mathrm{H} \gamma(300)$ & $04-08,08-16$ & $04-08,08-16$ & $04-08,11-13$ & $04-06,11-12$ & $04-06,11-12$ \\
\hline
\end{tabular}

${ }^{*}$ Region not defined for $T_{\text {eff }}=7500 \mathrm{~K}$.

\section{Conclusions and future work}

The measurement of accurate RVs of early-type (O-BA) stars cannot be achieved with the standard crosscorrelation techniques that are suitable for most latetype stars, where object-template spectrum mismatch and selection of wavelength range are not critical points. For early-type stars the occurrence of spectral-type mismatch commonly results in large systematic RV errors ("mismatch shifts") because their typically high rotational velocities cause spectral lines to blend into features that change shape with temperature and gravity, while their low line density prevents those errors from cancelling statistically.

In this first paper, we have used synthetic spectra in the range $3700-5200 \AA$ and with a resolution of $\sim 0.12 \AA$ per pixel to quantify the mismatch shifts that arise when differences between object and template spectrum of up to $\pm 500 \mathrm{~K}$ in $T_{\text {eff }}(\sim \pm 2$ temperature sub-classes $)$ and up to \pm 0.2 dex in $\log g(\sim \pm 1$ luminosity class $)$ are considered, representing 14 cases of spectral-type mismatch; variations in $v \sin i$ were not included as a source of mismatch. The mismatch shifts that were derived from our cross-correlations are valid only for spectra in which spectral features are well sampled. The expected mismatch error $E_{\mathrm{RV}}$, conservatively defined as the maximum mismatch shift that can occur for all 14 mismatch cases, was derived for each of 30 main-grid spectra whose range spanned the rotating A-type main-sequence stars $\left(T_{\text {eff }}=7500,8000\right.$, 8500,9000 and $9500 \mathrm{~K}, \log g=4.0$, and $v \sin i=5,50,100$, 150,200 and $300 \mathrm{~km} \mathrm{~s}^{-1}$ ). We derived mismatch errors for a set of several tens of spectral regions, five including $\mathrm{H}$ lines, in each spectrum.
We derive the following conclusions from our analyses of the mismatch errors of individual spectral regions in different spectra.

- A large range in mismatch errors arises between the different spectral regions for any given spectrum: the $10 \%$ highest errors are on average about a factor 20 larger than the $10 \%$ smallest ones;

- For the metal-line regions, the sign of the mismatch error is not randomly distributed in different spectral regions for many of the spectra with $v \sin i \geq 100 \mathrm{~km} \mathrm{~s}^{-1}$;

- Mismatch errors tend to increase monotonically with $v \sin i$ because blending becomes more severe with increasing rotational blurring. On average, mismatch errors scale linearly with $v \sin i$, but there is a large scatter owing to different degrees of individual blending;

- For a given spectral region, the mismatch errors may vary up to a factor 10 across the A-type temperature sequence. For most of the spectral regions, the variation of $E_{\mathrm{RV}}$ with temperature is monotonic but varies substantially in sign and in range from one region to the next, according to individual details of the spectrum mismatch. Average mismatch errors for the "best" regions, i.e. the ones with a weak temperature dependence and a small average $E_{\mathrm{RV}}$, are $\sim 0.04,0.4$, $1,1.5,2$ and $3 \mathrm{~km} \mathrm{~s}^{-1}$ for $v \sin i=5,50,100,150,200$ and $300 \mathrm{~km} \mathrm{~s}^{-1}$, respectively;

- Mismatch errors from H-line regions lie well within the same range as those from metal-line regions. Errors arising from those containing $\mathrm{H} \delta, \mathrm{H} \gamma$ and $\mathrm{H} \beta$ are relatively small for all spectra. The region containing $\mathrm{H} \epsilon$ yields relatively large mismatch errors in mid- and 
late-A spectra owing to blending with the $\mathrm{CaII} \mathrm{H}$ and $\mathrm{K}$ lines. The region containing $\mathrm{H} 14-\mathrm{H} 8$ yields worsening errors for late A-type spectra when $v \sin i \geq$ $100 \mathrm{~km} \mathrm{~s}^{-1}$.

Because statistical cancelling of errors is very inefficient, the mismatch error may increase substantially when more spectral regions are included in the cross-correlation. Accidental cancelling of large errors among individual spectral regions may also occur but will depend on spectral details that are not predictable in real spectra of individual stars. The development of a robust strategy for selecting individual spectral regions in order to minimize the mismatch error of the resulting combined region is therefore mandatory.

In view of the above considerations, we selected the different sets of spectral regions given in Table 2 for the 5 different ranges in $v \sin i$; they are suitable for the whole of the A-type main-sequence in each case. We made separate selections for the metal-line and for $\mathrm{H}$-line regions. The use of those selections meets a number of important requirements: accidental cancelling of large errors is avoided, sufficient spectral information is included, and the selection is uniform in $T_{\text {eff }}$ and continuous in $v \sin i$. For $v \sin i \leq$ $100 \mathrm{~km} \mathrm{~s}^{-1}$, the selection based on metal-line regions leads to smaller errors than that based on H-line regions. For $v \sin i>100 \mathrm{kms}^{-1}$, regions with $\mathrm{H}$ lines may be superior; the latter depends in practice on the additional mismatch arising from differences in spectrum rectification, a step which is especially cumbersome for echelle spectra (e.g. Verschueren et al. 1997 and references therein). Our wavelength selection yields expected maximum mismatch errors for all A-type main-sequence spectra of $\sim 0.05,0.15$, $0.5,1,1.5$ and $1.7 \mathrm{~km} \mathrm{~s}^{-1}$, respectively, for $v \sin i=5,50$, 100, 150, 200 and $300 \mathrm{kms}^{-1}$. To ensure random errors not exceeding those systematic errors, a $S / N$ of approximately 50-400 is required, depending on individual cases.

The size of the mismatch errors quoted above depends strongly on the size of the spectrum mismatch that we considered probable. If a half step in $T_{\text {eff }}$ has to be bridged, the mismatch errors will be approximately halved owing to the quasi-linear dependence of the mismatch shifts on $T_{\text {eff }}$ over that range. Decreasing the step in $\log g$ only becomes important if the mismatch error is no longer dominated by differences in $T_{\text {eff }}$. Detailed spectrum matching, using a dense grid of template spectra, therefore remains of key importance for increasing the RV accuracy. Expected errors may also be reduced by relaxing the requirement that one set of selected spectral regions must be suitable for the whole of the A-type temperature sequence. That will be a natural refinement as the present study is extended to include B- and O-type spectra.

We stress that the selection of spectral regions proposed here is based solely on a phenomenological study of synthetic spectra, and must therefore be regarded as tentative and subject to verification from studies with observed spectra. We already showed in Sect. 4 that this study confirms the suitability of the wavelength region used by Fekel $(1985,1999)$ for all A-type stars with $v \sin i$ $<50 \mathrm{~km} \mathrm{~s}^{-1}$, but at the same time hints at problems that will arise for faster rotators. The next paper, which will study real spectra of A-type stars, should enable us to gain insight into the possible breakdown of some of our conclusions under conditions of other types of mismatch, such as arise (for example) through differences in chemical abundances. By using real spectra, we will be forced to confront the complex inter-relationships between the many atmospheric parameters which the use of synthetic spectra has allowed us to handle as independent and controllable quantities, and to investigate the less well understood links between those parameters and others arising from other atmospheric variants not included in the model atmospheres. Future work will also investigate the identification of those spectral features that are chiefly responsible for large mismatch errors (and for the non-randomness of their sign). By combining those results with the technical experience gained in the present study, we hope eventually to be able to propose wavelength-selections that are firmly based on astrophysical arguments and which are likely to be workable in practice.

Acknowledgements. We are very grateful to Herman Hensberge for many enlightening discussions in the course of this project and for a critical reading of the manuscript. Myriam Vrancken is thanked for valuable help with the synthesis of the spectra. WV acknowledges financial support from the Fund for Scientific Research - Flanders (Belgium) (F.W.O.) through Research Grant No. 1.5.549.98. REMG is grateful to RUCA for the support of a Visiting Professorship.

\section{References}

Andersen J., Nordström B., 1983, A\&A 122, 23

Baranne A., Mayor M., Poncet J.L., 1979, Vistas Astron. 23, 279

Baranne A., Queloz D., Mayor M., et al., 1996, A\&AS 119, 373

Barnard A.J., Cooper J., Smith E.W., 1974, JQSRT 14, 1025

Collins II G.W., Truax R.J., 1995, ApJ 439, 860

David M., Verschueren W., 1995, A\&AS 111, 183

Dravins D., 1985, IAU Colloq. 88, Stellar Radial Velocities, Davis Philip A.G., Latham D.W. (eds.). L. Davis Press, NY, p. 311

Ebbets D., 1979, ApJ 227, 510

Fekel F.C., 1985, IAU Colloq. 88, Stellar Radial Velocities, Davis Philip A.G., Latham D.W. (eds.). L. Davis Press, NY, p. 335

Fekel F.C., 1999, IAU Colloq. 170, Precise Stellar Radial Velocities, Hearnshaw J.B., Scarfe C.D. (eds.). ASP Conf. Series (in press)

Hubeny I., Lanz T, Jeffery C.S., 1994, in Newsl. Anal. Astron. Spectra, No. 20, Jeffery C.S. (ed.). St. Andrews Univ., St. Andrews, p. 30

Kurucz R.L., 1979, ApJS 40, 1

Kurucz R.L., 1988, in Trans. of the IAU, Vol. XXB, McNally M. (ed.). Kluwer, Dordrecht, p. 168 
Kurucz R.L., 1993, S.A.O. CD-ROM 13

Kurucz R.L., Bell B., 1995, S.A.O. CD-ROM 23

Latham D.W., 1992, I.A.U. Colloq. 135, Complementary Approaches to Binary and Multiple Star Research, McAlister H., Hartkopf W. (eds.). ASP Conf. Ser. 32, 110

Latham D.W., Stefanik R.P., 1992, Trans. IAU Vol. XXIB, p. 269

Liu T., Janes K.A., Bania T.M., 1989, AJ 98, 626

Liu T., Janes K.A., Bania T.M., 1991a, ApJ 377, 141

Liu T., Janes K.A., Bania T.M., 1991b, AJ 102, 1103

Morse J.A., Mathieu R.D., Levine S.E., 1991, AJ 101, 1495

Nordström B., Latham D.W., Morse J.A., et al., 1994, A\&A 287, 338

Petrie R.M., 1962, in Astronomical Techniques, Hiltner W.A. (ed.). Univ. of Chicago Press, p. 63

Scarfe C.D., Batten A.H., Fletcher J.M., 1990, Publ. Dom. Astr. Obs. 18, No. 2, p. 21
Shamey L., 1969, Ph.D. Thesis, Univ. of Colorado

Stefanik R.P., 1997, Reports on Astronomy, Vol. XXIIIA, Appenzeller I. (ed.), IAU, p. 526

Verschueren W., 1991, Ph.D. Thesis, Free Univ. of Brussels (VUB), Belgium

Verschueren W., 1995, The Standard Star Newsletter 18, 6. Corbally C. (ed.)

Verschueren W., David M., 1999, A\&AS 136, 591

Verschueren W., Brown A.G.A., Hensberge H., et al., 1997, PASP 109, 868

Verschueren W., David M., Vrancken M., 1999, IAU Colloq. 170, Precise Stellar Radial Velocities, Hearnshaw J.B., Scarfe C.D. (eds.). ASP Conf. Ser. (in press)

Vidal C.R., Cooper J., Smith E.W., 1973, ApJS 25, 37

Vrancken M., Hensberge H., David M., Verschueren W., 1997, A\&A 320,878 